\title{
An Injection Method for Self-Calibration of Inductive Voltage Dividers
}

\author{
Wilbur C. Sze \\ Institute for Basic Standards, National Bureau of Standards, Washington, D.C. 20234 \\ (November 14, 1967) \\ A self-calibration or "boot-strapping" injection method for determining the voltage ratio and phase \\ angle deviations of inductive voltage dividers utilizes specially constructed transformers for step and \\ differential voltage injections without reference to the fundamental electrical units or standards. Con- \\ struction details of transformers, derivation of equations, and systematic evaluation of uncertainties \\ are presented. Calibration of certain ratios involving a combination of dial settings on several decades \\ can be obtained as readily as that of a single decade. The accuracy of the results obtainable at $10 \mathrm{kHz}$ \\ is within $2 \times 10^{-7}$ of input for deviations less than $10 \mathrm{ppm}$ of input and within $1 \times 10^{-6}$ for deviations \\ up to $100 \mathrm{ppm}$ of input. This same calibration technique can readily be used to cover a frequency range \\ from 1 to $20 \mathrm{kHz}$. \\ Key Words: Calibration, in-phase deviation, inductive voltage divider, measurements, quadrature \\ deviation, ratio calibration, transformer, two-stage voltage transformer, voltage di- \\ vider, voltage ratio.
}

\section{Introduction}

Since voltage ratio is a dimensionless quantity, one may reasonably expect that it can be accurately determined in any laboratory without reference to any standards or fundamental elec. trical units. It is also reasonable to assume that the limitations on the order of accuracy obtainable depend primarily on the knowledge and ingenuity of the developer and to a lesser extent on the commercially available equipment.

At the National Bureau of Standards, a new calibration method has been developed for determining the in-phase and quadrature deviations of inductive voltage dividers. The advantages of this technique are: (1) the test dividers may be calibrated as either three-terminal or fourterminal networks; (2) the test circuit imposes zero burden on the test divider; and (3) certain combinations of dial settings involving several decades can readily be calibrated.

The purpose of this paper is: (a) to describe a self-calibration or "boot-strapping" method; (b) to show the construction details of special transformers for step and differential voltage injections; and (c) to analyze various factors affecting the accuracy of the measurements. ${ }^{1}$ In this paper, the discussion is primarily centered at $10 \mathrm{kHz}$. However, the same method of calibration and technique of design can readily be used to cover a broad frequency range from 1 to $20 \mathrm{kHz}$.

\section{Inductive Voltage Divider Circuits}

Inductive voltage dividers are basically "three-terminal" devices; that is, they consist of two terminals for voltage input and an adjustable intermediate tap or output terminal. However, commercial type dividers are generally provided with two "common" terminals for convenience in

1 This work was supported in part by the Metrology Engineering Center, Bureau of Naval Weapons, Pomona, Calif. 
making connections (fig. 1). The voltage differences between the "common" terminals in such "four-terminal" devices, due to internal pickup and impedance of the connecting wire, may be significantly large at high operating frequencies and may vary with the settings of the dials. In order to prevent the introduction of electrostatically induced voltages into the connecting lead, a copper shield has been added over it in the dividers used in this study. In figure 3 , this shield and the case of the divider are electrically connected to the "common" terminal located near the "input" terminal. ${ }^{2}$ The maximum measured voltage difference $\alpha_{l C x}^{\prime \prime}+j \beta^{\prime \prime} \widetilde{C} x$ (fig. 3) is reduced by addition of the shielding to the connecting wire from about $5 \times 10^{-7}$ to less than $3 \times 10^{-8}$ of the input voltage. Further reduction can be achieved by shielding the output terminals (i.e., replacing them with coaxial type).

It is not necessary to add the shield in order to calibrate the divider with the method described here. However, it is a convenience in the calibration procedure particularly if the divider is to be used as a reference standard for calibration of other working dividers.

Throughout the discussion in this paper, the "common" terminal located near the "input" terminal will be disignated as the "reference" point for all voltage measurements, see figure 3 .

\section{Method of Measurement}

\subsection{Description of Circuit}

Figure 2 shows the schematic circuit diagram simplified by omission of the guard circuits and shielding arrangements. $D_{1}$ and $D_{2}$ represent the test inductive voltage dividers whose in-phase and quadrature deviations are being determined. $\mathrm{D}_{1}$ and $\mathrm{D}_{2}$ can be calibrated simultaneously or one can be treated as an auxiliary. Their input terminals are connected together with a short coaxial lead, the impedances of which, from the junction point to the input terminals of each divider, must be very small relative to the divider input impedances. Their output "common" terminals are connected together with a low resistance lead.

The calibration circuit uses three toroidal transformers: one transformer for injection of step voltage, the second for injection of differential voltage, and a third for null detection.

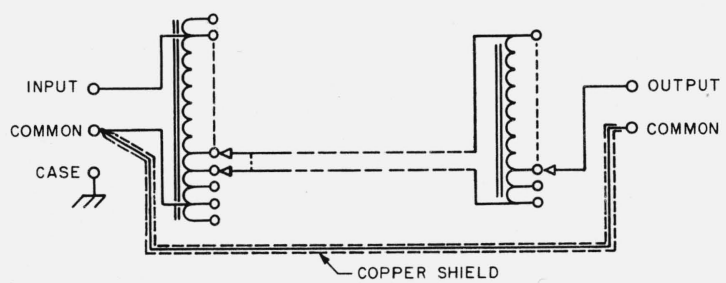

Figure 1. Typical "four-terminal" divider circuit (shown with copper shield added by NBS).

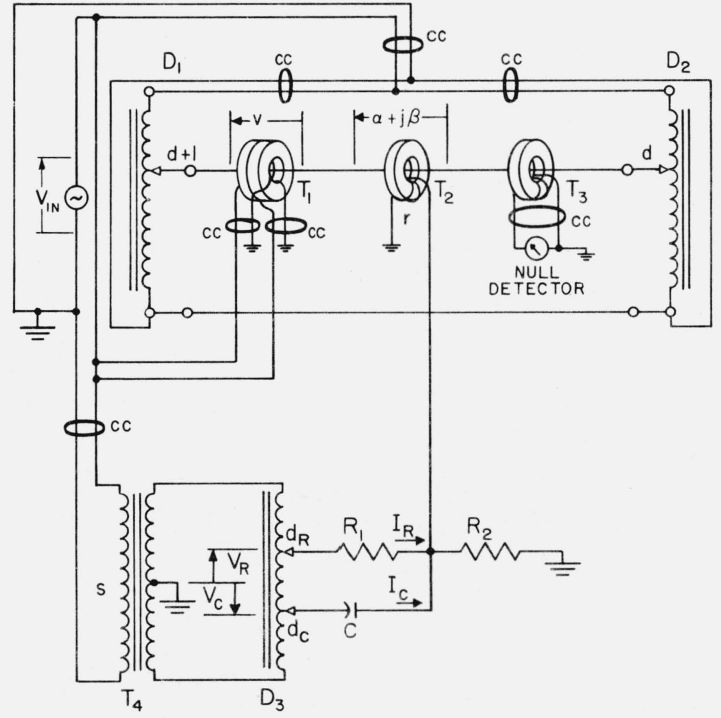

FigURE 2. Schematic circuit diagram (simplified by omission of the shielding arrangements and guard circuits).

"On some commercial dividers, the case ground terminal is located near the "output" terminal. A suitable lead may then be used to connect the case to the "common" terminal located near the "input" terminal. 
Transformer $T_{1}$ (each primary winding is represented by one-turn in figure 2 for simplicity) injects a voltage, $v$ (approximately equal to that corresponding to one step of the decade being calibrated) into the lead connecting the "tap" output terminals of the two dividers. When $\mathrm{T}_{1}$ is energized, the tap setting, $d+1$, of divider $\mathrm{D}_{1}$ is set one step higher than the tap setting, $d$, on the corresponding decade of divider $D_{2}$. Inductive voltage divider $D_{3}$, injects, through the R-C network and transformer $\mathrm{T}_{2}$, in-phase and quadrature differential balancing voltages, $\alpha+j \beta$, in the same connecting lead.

The detector circuit consists of a tuned null-indicator and a specially constructed shielded transformer, $T_{3}$, to match the impedance and to isolate the detector from the measuring circuit.

Transformer, $\mathrm{T}_{4}$, is also specially constructed with an accurate voltage ratio, $s$, of either 1:1 or 1:2. The midpoint of the secondary is grounded so that either polarity can be obtained from the outputs of $D_{3}$ without the necessity of altering the circuit.

\subsection{Calibration Procedure}

When calibrating commercial type inductive voltage dividers which have only one adjustable output terminal, two such dividers, shown as $D_{1}$ and $D_{2}$ in figures 2 and 3 , must be used. The procedure is as follows: First, determine the voltage, $\alpha_{n}^{\prime \prime}+j \beta_{n}^{\prime \prime}$, corresponding to balance with both dividers at a given setting, $d$, with the transformer $\mathrm{T}_{1}$ deenergized; second, determine the balancing voltage, $\alpha_{n}^{\prime}+j \beta_{n}^{\prime}$, required with $\mathrm{T}_{1}$ energized and the tap setting on divider $\mathrm{D}_{1}$ one step higher. Repeat these two measurements on each of the ten settings to provide a "boot-strapping" calibration of the decade.

\subsection{Coaxial Chokes}

In order to realize the desired accuracy, coaxial chokes are required in various branches of the circuit. These reduce the small but troublesome loop and ground currents to insignificant values. The use of coaxial chokes in related applications has been discussed by Thompson, Homan, and Cutkosky [1, 2, 3, 4]. ${ }^{3}$ Each coaxial choke consists of an appropriate number of turns of a coaxial cable threaded through a high permeability toroidal core, located and shown as "cc" in figures 2 and 4. The operating principle is as follows: When the currents in the outer and center conductors of a coaxial lead are equal but in opposite directions, no flux is generated in the core. If either a loop, pickup, or leakage current causes an unequal flow of currents in the coaxial lead, a flux is generated in the core which in turn produces a high impedance in the particular path. Thus the undesirable current is reduced to a negligible value.

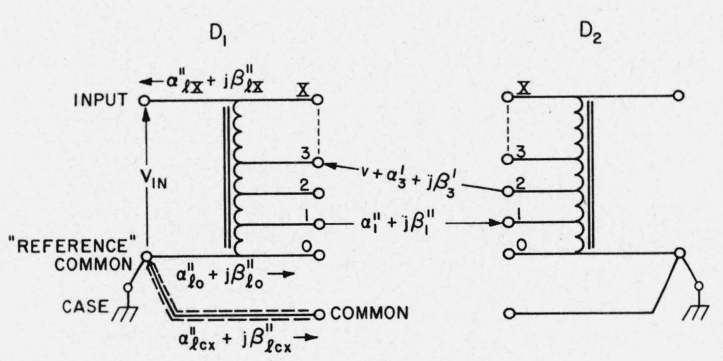

FIGURE 3. Circuit for self "boot-straping" calibration.

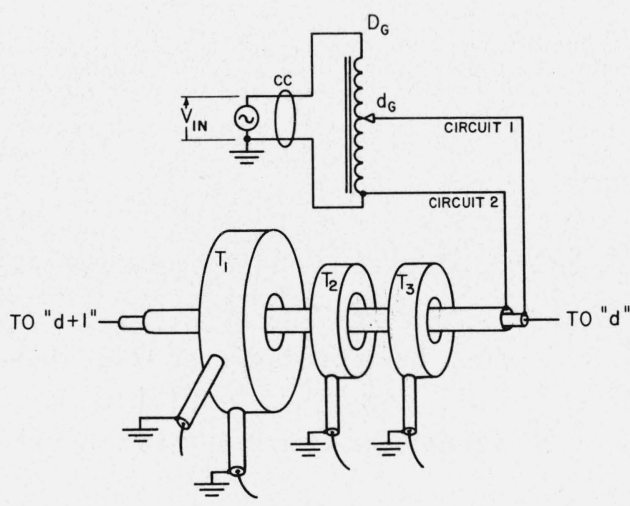

FIGURE 4. Guard circuit. 


\subsection{Guard Circuits}

It is imperative to the success of this "boot-strapping" method that the step-voltage injector transformer, $\mathrm{T}_{1}$, maintain precisely the same value of voltage, $v$, in its secondary winding regardless of the dial settings of the test dividers. This requirement necessitates that the lead connecting the output terminals of the test dividers be doubly guarded as shown in figure 4 , to eliminate errors from capacitance currents.

A guard inductive voltage divider, $\mathrm{D}_{\mathrm{G}}$, is used to supply the voltages of the guard circuits. Circuit 1 is connected to the "tap" output terminal of the guard divider, with its dials set corresponding to that of the adjacent test divider (i.e., $d=d_{\mathrm{i}} \neq d+1$ ). Circuit 2 is connected to the "common" output terminal which is at ground potential. Thus the voltage between the center conductor and circuit $l$ is approximately zero regardless of the dial settings on the test dividers, and no significant capacitive current should exist. The same is true between the cases of transformers $\left(\mathrm{T}_{1}, \mathrm{~T}_{2}\right.$, and $\mathrm{T}_{3}$ ) and circuit 2 . The leakage current between circuits 1 and 2 constitutes a variable load on the guard divider which is independent of the measurement circuits.

A voltage difference approximately equal to $v$ exists between the case of transformer $\mathrm{T}_{1}$ and that portion of the circuit 2 between it and the "tap" output terminal of divider $\mathrm{D}_{1}$. The leakage current constitutes a load on $\mathrm{T}_{1}$. However, this leakage remains at a constant value throughout the "boot-strapping" test procedure and does not affect the test results.

\section{Theoretical Relations}

By definition, the voltage ratio of a divider is the ratio of the output terminal voltage, $V_{\text {out }}$, to the input terminal voltage, $V_{\text {in }}$, which can be expressed as

$$
\frac{V_{\text {out }}}{V_{\text {in }}}=d+a+j b
$$

where $d$ is the nominal ratio or setting of the dials, $a$ is the in-phase deviation, and $b$ is the quadrature deviation. Both deviations are expressed as fractions of input or unity.

\subsection{Differential Injection Voltage, $\alpha+j \beta$}

The equations for the in-phase and quadrature differential injection voltages, $\alpha+j \beta$, will be derived first from the $\mathrm{R}-\mathrm{C}$ network in figure 2 as follows:

$$
\begin{aligned}
& V_{R}=I_{R} R_{1}+r(\alpha+j \beta), \\
& V_{C}=-I_{C}\left(j \frac{1}{\omega C}\right)+r(\alpha+j \beta),
\end{aligned}
$$

and

$$
r(\alpha+j \beta)=\left(I_{C}+I_{R}\right) \frac{R_{2} Z}{R_{2}+Z} \approx\left(I_{C}+I_{R}\right) R_{2}
$$

where $Z$ is the impedance of transformer $\mathrm{T}_{2}$, referred to its primary side, and $r$ represents its voltage ratio. Since the circuit described here, $Z \gg R_{2}$, the above approximation is justified.

By solving for $I_{R}$ and $I_{C}$ from the first two equations and substituting in the third,

$$
r(\alpha+j \beta)=\frac{V_{C} R_{1}^{2}+V_{R}\left(\frac{1}{\omega C}\right)^{2}\left(1+\frac{R_{1}}{R_{2}}\right)+j R_{1}\left(\frac{1}{\omega C}\right)\left[V_{C}\left(1+\frac{R_{1}}{R_{2}}\right)-V_{R}\right]}{R_{1}^{2}+\left(\frac{1}{\omega C}\right)^{2}\left(1+\frac{R_{1}}{R_{2}}\right)^{2}} .
$$


It is convenient to express $V_{C}, V_{R}$, and $\alpha+j \beta$ on a per-unit-input basis. $V_{C} / V_{\text {in }}$ and $V_{R} / V_{\text {in }}$ may be expressed as $\frac{1}{s}\left(d_{C}-0.5\right)$ and $\frac{1}{s}\left(d_{R}-0.5\right)$, respectively, where $d_{C}$ and $d_{R}$ are dial settings of divider $\mathrm{D}_{3}$ and $s$ represents the voltage ratio of $\mathrm{T}_{4}$. (The necessity for subtracting 0.5 from the dial readings of $D_{3}$ arises from the fact that the center tap of the secondary winding of $T_{4}$ is grounded.) Then by multiplying the above equation by $p$ and separating real and imaginary terms,

$$
\begin{aligned}
& p \alpha=\frac{1}{s r}\left[\frac{\left(d_{C}-0.5\right) R_{1}^{2}+\left(d_{R}-0.5\right)\left(\frac{1}{\omega C}\right)^{2}\left(1+\frac{R_{1}}{R_{2}}\right)}{R_{1}^{2}+\left(\frac{1}{\omega C}\right)^{2}\left(1+\frac{R_{1}}{R_{2}}\right)^{2}}\right] \approx\left(d_{R}-0.5\right)\left(\frac{R_{2}}{s r R_{1}}\right) \\
& p \beta=\frac{1}{s r}\left[\frac{\frac{R_{1}}{\omega C}\left[\left(d_{C}-0.5\right)\left(1+\frac{R_{1}}{R_{2}}\right)-\left(d_{R}-0.5\right)\right]}{R_{1}^{2}+\left(\frac{1}{\omega C}\right)^{2}\left(1+\frac{R_{1}}{R_{2}}\right)^{2}}\right] \approx\left(d_{C}-0.5\right)\left[\frac{R_{2}}{s r\left(\frac{1}{\omega C}\right)}\right]
\end{aligned}
$$

where $p=1 / V_{\text {in }}$.

The above approximations are justified ${ }^{4}$ since $\frac{1}{\omega C} \simeq R_{1}$ and $R_{1} \gg R_{2}$.

\subsection{In-Phase and Quadrature Deviations, $a+j b$}

In figure $3, V_{\text {in }}$ represents the input terminal voltage, $v$ the step-injection voltage of transformer $\mathrm{T}_{1} ; \alpha_{n}^{\prime}+j \beta_{n}^{\prime}$ and $\alpha_{n}^{\prime \prime}+j \beta_{n}^{\prime \prime}$ the small balancing voltages from transformer $\mathrm{T}_{2} ; \alpha_{l 0}^{\prime \prime}+j \beta_{l 0}^{\prime \prime}$ the voltage drop between the "reference" terminal and the "tap" output terminal when the dial of the decade under test is set on 0 ; and $\alpha_{l X}^{\prime \prime}+j \beta_{l X}^{\prime \prime}$ the voltage drop between the "high" input terminal and the "tap" output terminal when the dial is set on $X$. The voltage drop, $\alpha_{l C x}^{\prime \prime}+j \beta_{l C x}^{\prime \prime}$, measured between the "common" terminals in general depends on the dial settings. The subscript $x$ identifies the dial setting. Then from figure 3 (note assigned voltage polarities), the equation for a four-terminal voltage ratio is derived as follows:

$$
V_{\mathrm{in}}=\alpha_{l 0}^{\prime \prime}+j \beta_{l 0}^{\prime \prime}+10 v+\sum_{n=1}^{10} \alpha_{n}^{\prime}+\sum_{n=1}^{10} j \beta_{n}^{\prime}+\sum_{n=0}^{9} \alpha_{n}^{\prime \prime}+\sum_{n=0}^{9} j \beta_{n}^{\prime \prime}+\alpha_{l X}^{\prime \prime}+j \beta_{l X}^{\prime \prime}
$$

and the voltage ratio for any setting $x$ on the top decade can be expressed as

$$
\begin{aligned}
\frac{V_{\text {out }}}{V_{\text {in }}}= & \frac{\alpha_{l 0}^{\prime \prime}+j \beta_{l 0}^{\prime \prime}-\alpha_{l C x}^{\prime \prime}-j \beta_{l C x}^{\prime \prime}+x v+\sum_{n=1}^{x} \alpha_{n}^{\prime}+\sum_{n=1}^{x} j \beta_{n}^{\prime}+\sum_{n=0}^{x-1} \alpha_{n}^{\prime \prime}+\sum_{n=0}^{x-1} j \beta_{n}^{\prime \prime}}{V_{\text {in }}} \\
= & \frac{\frac{x}{10}+\left(\alpha_{l 0}^{\prime \prime}+j \beta_{l 0}^{\prime \prime}-\alpha_{l C x}^{\prime \prime}-j \beta_{l C x}^{\prime \prime}+\sum_{n=1}^{x} \alpha_{n}^{\prime}+\sum_{n=1}^{x} j \beta_{n}^{\prime}+\sum_{n=0}^{x-1} \alpha_{n}^{\prime \prime}+\sum_{n=0}^{x-1} j \beta_{n}^{\prime \prime}\right) \frac{1}{10 v}}{1+\left(\alpha_{l 0}^{\prime \prime}+j \beta_{l 0}^{\prime \prime}+\sum_{n=1}^{10} \alpha_{n}^{\prime}+\sum_{n=1}^{10} j \beta_{n}^{\prime}+\sum_{n=0}^{9} \alpha_{n}^{\prime \prime}+\sum_{n=0}^{9} j \beta_{n}^{\prime \prime}+\alpha_{l X}^{\prime \prime}+j \beta_{l X}^{\prime \prime}\right) \frac{1}{10 v}}
\end{aligned}
$$

${ }^{4}$ Except for some change in notation, the above analysis has been presented elsewhere [5]. 
Since $10 v \simeq V_{\text {in }}$ for the top decade, and with $\frac{1}{V_{\text {in }}}=p$ as previously defined, a rearrangement of terms leads to $\frac{V_{\text {out }}}{V_{\text {in }}}=d+a+j b$

$$
\begin{aligned}
& \approx \frac{x}{10}+\left[p \alpha_{l 0}^{\prime \prime}-p \alpha_{l C x}^{\prime \prime}+\sum_{n=1}^{x} p \alpha_{n}^{\prime}+\sum_{n=0}^{x-1} p \alpha_{n}^{\prime \prime}-\frac{x}{10}\left(p \alpha_{l 0}^{\prime \prime}+\sum_{n=1}^{10} p \alpha_{n}^{\prime}+\sum_{n=0}^{9} p \alpha_{n}^{\prime \prime}+p \alpha_{l X}^{\prime \prime}\right)\right] \\
& \quad+j\left[p \beta_{l 0}^{\prime \prime}-p \beta_{l C x}^{\prime \prime}+\sum_{n=1}^{x} p \beta_{n}^{\prime}+\sum_{n=0}^{x-1} p \beta_{n}^{\prime \prime}-\frac{x}{10}\left(p \beta_{l 0}^{\prime \prime}+\sum_{n=1}^{10} p \beta_{n}^{\prime}+\sum_{n=0}^{9} p \beta_{n}^{\prime \prime}+p \beta_{l X}^{\prime \prime}\right)\right]
\end{aligned}
$$

If three-terminal voltage ratios are desired, the term $\alpha_{l C x}^{\prime \prime}+j \beta_{l C x}^{\prime \prime}$ is omitted from the above equations, and the "common" output terminal is ignored..$^{5}$

The foregoing derivation is for determining the voltage ratios of the top decade of divider $D_{1}$. By addition of a series of measurements of the internal voltage drops of divider $D_{2}$, a similar expression can be derived for determining the voltage ratios of $\mathrm{D}_{2}$.

For calibration of lower decades, using step-voltage injection transformers of appropriate ratios, similar equations can be derived.

\section{Description of Components}

$\mathrm{T}_{1}, \mathrm{~T}_{2}, \mathrm{~T}_{3}$, and $\mathrm{T}_{4}$ are specially constructed shielded transformers using high magnetic permeability strip wound toroidal cores to insure close magnetic coupling and to conserve space in the circuit. The shields minimize the susceptibility to magnetic pickup from stray fields in the laboratory.

The two-stage step-injection voltage transformer $T_{1}$ is shown in detail in figure 5 . This transformer injects a voltage $v$ which is closely equal to that of one step of the test decade. The principle involves a double circuit: one to provide a rough voltage and the other to correct it. The theory of operation is discussed by Cutkosky [6]. In figure 5, the voltage applied to winding 1 can be thought of as a rough generator which supplies excitation current to the transformer to obtain an approximation of the desired voltage ratio. The voltage applied to winding 2 then corrects this approximate voltage ratio. The number of turns for windings 1 and 2 is governed by the decade of the divider to be calibrated (i.e., 10 turns for the top or $10^{-1}$ decade; 100 turns for the $10^{-2}$ decade, etc.). Each winding is wound in a single, equally distributed layer from $0^{\circ}$ to $360^{\circ}$, and the wire is brought along the core from $360^{\circ}$ back to the point of origin. Thus no loop is formed, and

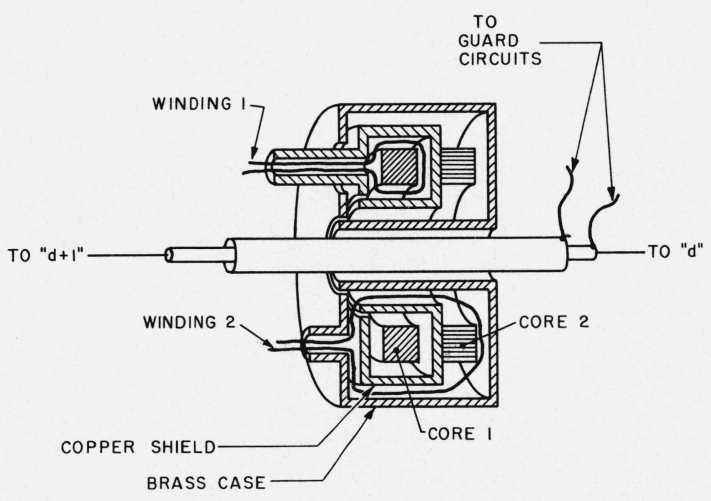

FigURE 5. Two-stage step-injection voltage transformer " $T_{1}$ ".

${ }^{5} \alpha_{l C x}^{\prime \prime}+j \beta_{l C x}^{\prime \prime}$ is measured with transformers, $\mathrm{T}_{2}$ and $\mathrm{T}_{3}$, connected between the "common" terminals in figure 1. 
susceptibility to stray magnetic pickup is greatly reduced. (This same technique is applied in winding all other transformers.) One-mil $(0.025 \mathrm{~mm})$ thick supermalloy-type strip toroidal cores of $38.1 \mathrm{~mm} \mathrm{ID}, 63.5 \mathrm{~mm} \mathrm{OD}$, and $12.7 \mathrm{~mm} \mathrm{H}$ are used. A copper eddy current shield 20 -mil $(0.51 \mathrm{~mm})$ thick between windings 1 and 2 reduces the stray magnetic fields in the region of winding 2 caused by excitation current in winding 1 , and hence improves the accuracy of the voltage ratio. The brass case is for electrostatic shielding.

Figure 6 shows the details of construction of transformers $T_{2}$ and $T_{3}$ as a single unit. They are identical and interchangeable. Each consists of a 100-turn primary winding on a 1-mil $(0.025 \mathrm{~mm})$ thick supermalloy-type strip toroidal core of $38.1 \mathrm{~mm} \mathrm{OD,} 25.4 \mathrm{~mm} \mathrm{ID}$, and $6.4 \mathrm{~mm} \mathrm{H}$. The shielding arrangements are such that the cross capacitances at the gaps are reduced to a minimum [7]. However, if the technique were to be used for calibration of dividers with large in-phase and quadrature voltage deviations which would require a significantly higher value for $R_{2}$, the crosssectional area of the core of the transformer designated as $T_{2}$ (differential voltage injection) would have to be increased, so that the current through the primary of $T_{2}$ would remain insignificant in comparison with that through $\mathrm{R}_{2}$ (i.e., $Z \gg \mathrm{R}_{2}$ ).

Figure 7 shows the construction of transformer $\mathrm{T}_{4}$. It consists of a toroidal core of the same dimensions as that used for $\mathrm{T}_{1}$, a secondary winding which is sandwiched between two copper eddy-current shields, two parallel sections (100-turns each) of primary winding, and an overall Mu-metal case. The sandwich design improves the accuracy of the voltage ratio. The midpoint of the secondary winding is grounded. Two versions of $\mathrm{T}_{4}$ have been made, one with a 100-turn secondary and the other with a 200-turn secondary, in order that voltage ratios, $s$, of $1: 1$ and $1: 2$ can be selected.

The inductive voltage divider, shown as $\mathrm{D}_{3}$ in figure 2 , actually consists of two commercial type units in parallel, one for in-phase voltage injection and the other for quadrature voltage injection. Each consists of three inductive decades and a continuously adjustable resistive divider.

$C$ is a three-terminal capacitor having a very small dissipation factor. $R_{1}$ and $R_{2}$ are threeterminal, low-reactance decade resistors of $10 \times 1 \mathrm{k} \Omega$ and $10 \times 10 \Omega$, respectively. For $10 \mathrm{kHz}$ calibrations, if an air capacitor of $1592 \mathrm{pF}$ is used for $C, s=1: 1, R_{1}=10 \mathrm{k} \Omega$, and $R_{2}=10 \Omega$, one step on the top decades of $D_{3}$ represents a difference of 1 ppm-in in in-phase or quadrature deviation. ${ }^{6}$

The detector circuit consists of a preamplifier and a cathode-ray oscillograph. A narrow band-pass filter is used to discriminate against noise before the first stage of amplification. A double elliptical pattern, suggested by Clothier, is shown on the oscillograph to indicate balance condition [8]. The unbalance voltage from the calibration circuit is impressed on the vertical plates. On the horizontal plates is placed a signal of the same frequency, whose phase can be adjusted so that the angular departure of the major axis of the elliptical pattern represents in-phase

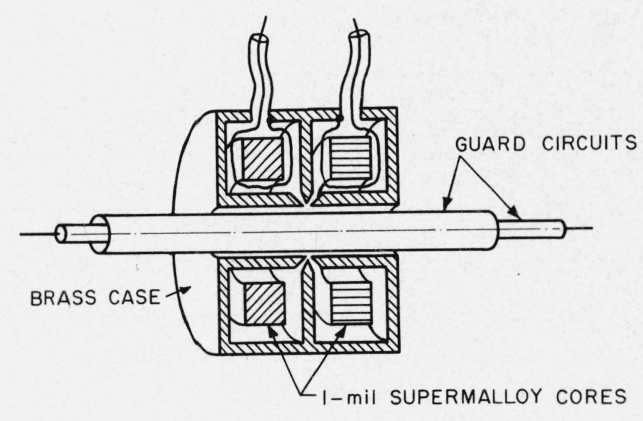

FIGURE 6. Injection-detection transformers " $T_{2}$ " and " $T_{3}$ ".

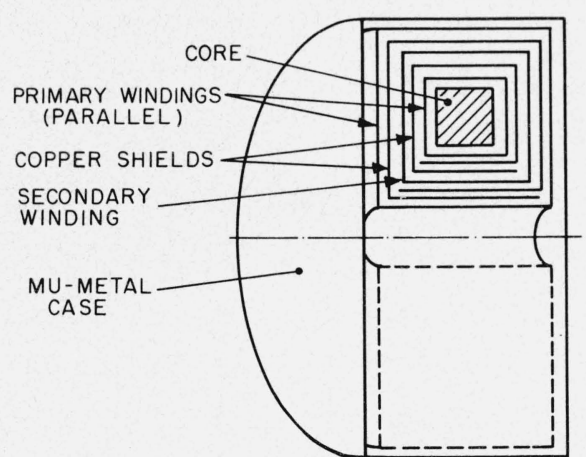

Figure 7. Shielded transformer " $T_{4}$ ".

${ }^{6} \mathrm{ppm}$-in (parts-per-million of input or unity). 
unbalance and opening along the minor axis quadrature unbalance. At balance, the figure appears to be a single line. An unbalance voltage of $100 \mathrm{pV}$ is easily observed.

\section{Ratios Involving a Combination of Several Decades}

For simplicity of presentation, sections 3 to 5 are written for the calibration of an individual decade. However, one may readily apply the same technique to calibration of voltage ratios involving a combination of dial settings on several decades of the test divider.

The turns ratio of the 2-stage step-injection voltage transformer, $\mathrm{T}_{1}$, determines the voltage $v$ (see figs. 2 and 5) which in turn governs the size of the step interval of the dial settings of the test dividers; i.e., if windings 1 and 2 were to consist of 40 turns each, the turns ratio would be $40: 1$, and the interval of the dial setting, 0.025 . Forty separate balances of $\alpha_{n}^{\prime}+j \beta_{n}^{\prime}$ and of $\alpha_{n}^{\prime \prime}+j \beta_{n}^{\prime \prime}$ would then provide a "boot-strapping" calibration at dial settings of $0.025,0.05,0.075$, etc.

\section{Two-Stage Multitap Single-Range Dividers}

The claimed accuracy of results has been verified by construction of two-stage single-range dividers, whose taps are all brought out to external connectors. Their construction is similar to the voltage transformer shown in figure 5 , but both guard circuits and the center conductor are omitted, and a Mu-metal shield substituted for the copper shield. Figure 8 shows the arrangement of external connectors on a 20 -section and a 40 -section divider.

For the 20-section divider, winding 1 (fig. 5) consists of 100 turns and winding 2 of 5 turns of a 20-wire cable. The wires in the cable are connected in series, and the junctions are made at the connectors on the cover. The 2 -stage 40 -section is similarly constructed.

Since a single divider can be self-calibrated providing all the taps are brought out externally, $D_{1}$ and $D_{2}$ of figure 2 can be thought of as a single divider. The in-phase and quadrature voltage deviations of these two special dividers were obtained with both 10:1 and 20:1 voltage ratio step-injection transformers, $\mathrm{T}_{1}$, and the 40 -section divider was also calibrated with a $40: 1$ voltage ratio $\mathrm{T}_{1}$.

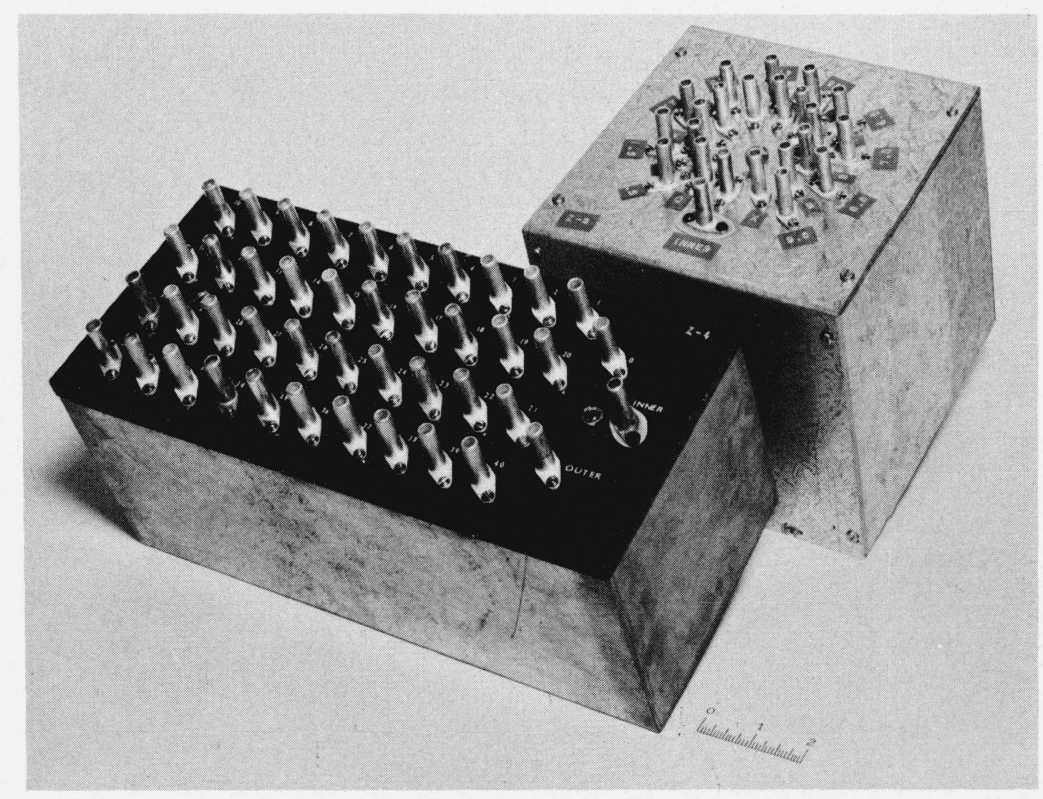

FIGURE 8. Two-stage single-range dividers. 
Since the high input terminal and the highest output terminal are located immediately adjacent to one another and are connected together with a very short copper bus, and the same is true for the low input and " 0 " output terminals, the voltage differences, $\alpha_{l 0}^{\prime \prime}+j \beta_{l 0}^{\prime \prime}$ and $\alpha_{l X}^{\prime \prime}+j \beta_{l X}^{\prime \prime}$ are thus reduced to zero. Therefore, the calibration procedure required obtaining $\alpha_{n}^{\prime}+j \beta_{n}^{\prime}$ balances only.

These single range dividers were: (1) further calibrated in combination with commerical type dividers in the self-calibration circuitry and (2) used for comparison calibration in the comparator circuitry [5] to verify that the accuracy of measurements obtainable by the self-calibration or "boot-straping" technique.

The results obtained on approximately 1300 separate measurements are too voluminous to show here. In general, about 80 percent of the measurements agreed to within $1 \times 10^{-7} \mathrm{ppm}$-in, while the lack of agreement exceeded $2 \times 10^{-7} \mathrm{ppm}$-in in only about 2 percent of the approximately 1300 separate measurements. These close agreements also indicated that the method of measuring $\alpha_{l 0}^{\prime \prime}+j \beta_{l 0}^{\prime \prime}, \alpha_{l X}^{\prime \prime}+j \beta_{l X}^{\prime \prime}$, and $\alpha_{l C x}^{\prime \prime}+j \beta_{l C x}^{\prime \prime}$ as outlined previously is correct.

\section{Consideration of Errors}

An examination of uncertainties requires a systematic evaluation of all possible errors and a careful appraisal of all components of the measuring circuit as well as a justification of estimations, even though in this case one would expect most of the associated errors and uncertainties to be negligible.

The first source of errors to be considered is the differential voltage injection circuit. Approximations are made in developing the expressions for obtaining the in-phase and quadrature deviations, $\alpha+j \beta$, where the effects of the residual of the decade resistors and the dissipation factor of the capacitors are ignored. Approximate magnitudes of these errors have been obtained by adding the estimated equivalent residual reactance across $R_{1}$ and $R_{2}$ and estimated series resistance to $C$. These errors have been found to be negligible.

Another justifiable approximation is omitting the impedance, $Z$, of transformer $\mathrm{T}_{2}$ from the expression for $\alpha+j \beta$. Since $Z \approx 2.5 \times 10^{4} \mathrm{ohms}$ and $R_{2}=10 \Omega$, the current through $Z$ is relatively insignificant. Calibration (with appropriate output loading) of the pair of inductive voltage dividers, $\mathrm{D}_{3}$, introduces another source of uncertainty. However, experience indicated that errors in them should not exceed $0.5 \mathrm{ppm}$-in. Uncertainties in obtaining the voltage ratio correction due to excitation and variable load currents of the transformers $\mathrm{T}_{2}$ and $\mathrm{T}_{4}$ should not exceed $0.5 \mathrm{ppm}$ of indicated ratio. However, since the injection voltage, $\alpha+j \beta$, rarely exceeds 0.002 percent of the input voltage, the overall uncertainty, including the errors caused by the associated leads, contacts, and imperfect shields, is estimated to be $<0.05$ ppm-in.

The second source to be considered is the step-voltage injection circuit. As indicated before, it is imperative that the injection voltage, $v$, of $\mathrm{T}_{1}$ be constant throughout the "boot-strapping" calibration. This requires an extensive guard arrangement. Since both guard circuits and the lead connecting the "tap" output terminals constitute the secondary windings of $T_{1}$, any leakage current is a secondary load on $T_{1}$. The uncertainties from this source are determined as outlined in section 7 to be $<0.06$ ppm-in.

The third and largest source of uncertainty to be appraised is the guard arrangement. The primary function of the guard circuits is to intercept or eliminate any leakage currents, and their secondary function is to prevent electrostatic pickups. Because of the nature of the terminals used on the commercial test dividers, the shields at either end are not as complete as they could be; and because neither a detector nor a quadrature voltage is used to balance the voltage between guard circuit 1 and the center conductor, a small leakage current exists. These errors are estimated to be about $0.08 \mathrm{ppm}$-in for in-phase and $0.10 \mathrm{ppm}$-in for quadrature deviations.

A significantly large error is inherent in obtaining the measurements $\alpha_{l C x}^{\prime \prime}+j \beta_{l C x}^{\prime \prime}$ if the internal lead connecting the "common" terminals is not shielded. Proper shielding as indicated in section 2 should reduce the uncertainty to $<0.01 \mathrm{ppm}$-in. 
The last to be considered are miscellaneous errors from all other sources not covered previously, such as pickup from stray fields in the laboratory, harmonics in power supply, loop currents in the circuits, lead resistance, contacts, and imperfect shieldings. These uncertainties are estimated to be $<0.05$ and $<0.07 \mathrm{ppm}$-in for in-phase and quadrature deviations, respectively.

Finally, an appropriate multiple of the standard deviation of the results obtained in repeated calibrations should be added to the combination of the systematic errors estimated above $[9,10]$.

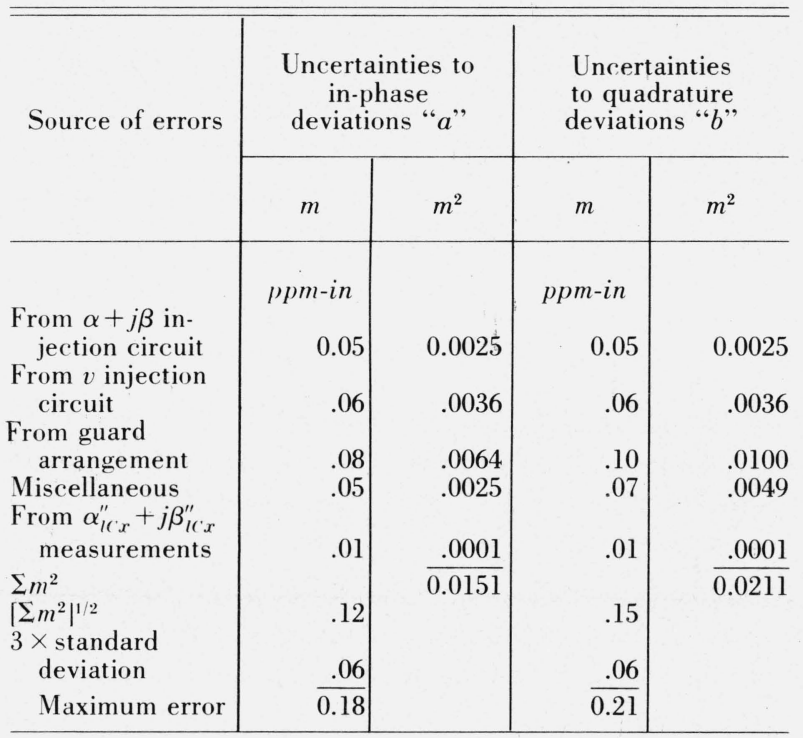

The maximum error values indicated in the table are for " $a$ " and " $b$ " deviations of $<10 \mathrm{ppm}$-in.

\section{Conclusion}

A high-accuracy self-calibration method has been developed for measuring the in-phase and quadrature voltage deviations of inductive voltage dividers at $10 \mathrm{kHz}$. An overall uncertainty within $2 \times 10^{-7}$ parts of input is achieved for dividers with deviations of less than $1 \times 10^{-5}$ parts of input, and $1 \times 10^{-6}$ is attainable for deviations up to $1 \times 10^{-4}$ parts of input.

This same calibration technique can readily be used to cover a frequency range from 1 to $20 \mathrm{kHz}$.

These high accuracy measurements are made without reference to any standards or fundamental electrical units.

Calibration of voltage ratios involving certain combinations of dial settings on several decades can be obtained as readily as on a single decade.

The author acknowledges the valuable help and suggestions received from Robert D. Cutkosky, Absolute Electrical Measurements Section, NBS. R. J. Berry contributed skillfully in constructing the components.

\section{References}

[1] The precision measurement of transformer ratios, R. D. Cutkosky and J. Q. Shields, IRE Trans. Instr. I-9, No. 2, 243-250 (Sept. 1960).

[2] The precise measurement of small capacitances, A. M. Thompson, IRE Trans. Instr. I-7, Nos. 3 and 4, 245-253 (Dec. 1958). 
[3] A.C. bridge methods for the measurement of three-terminal admittances, A. M. Thompson, IEEE Trans. Instr. Measr. IM-13, No. 4, 189-197 (Dec. 1964).

[4] Applications of coaxial chokes to ac bridge circuits, D. N. Homan, in press.

[5] Comparator for calibration of inductive voltage dividers from 1 to $10 \mathrm{kHz}$, Wilbur C. Sze, ISA 1966 Conference, Paper No. 12.3-2-66, October 24-27, 1966, New York.

[6] Active and passive direct-reading ratio sets for the comparison of audio-frequency admittances, R. D. Cutkosky, J. Res. NBS 68C (Engr. and Instr.) No. 4, 227-236 (Oct.-Dec. 1964).

[7] The NBS gas thermometer II. Measurements of capacitance to a grounded surface with a transformer ratio-arm bridge,

L. A. Guildner and R. E. Edsinger, J. Res. NBS 69C (Engr. and Instr.) No. 1, 13-18 (Jan.-Mar. 1965).

[8] A cathode-ray oscilloscope bridge balance display, W. K. Clothier, J. Sci. Instr. 43, pp. 264-265 (April 1966).

[9] An analysis of errors in the calibration of electrical instruments, F. L. Hermach, AIEE Trans. Comm. and Elctn., Paper 61-63, May 1961.

[10] Experimental statistics, M. G. Natrella, NBS Handbook 91, Chap. 23, Aug. 1, 1963.

(Paper 72C1-268) 